\title{
Stator Short Circuits Detection in PMSM by means of Higher Order Spectral Analysis (HOSA)
}

\author{
J. Rosero ${ }^{1}$, J. Ortega ${ }^{2}$, J. Urresty ${ }^{2}$ J. Cárdenas ${ }^{2}$, L. Romeral ${ }^{2}$ \\ ${ }^{1} \mathrm{ABB}$, e-mail: javier.rosero@es.abb.com \\ ${ }^{2}$ Motion Control and Industrial Applications Group, Technical University of Catalonia, \\ C/ Colon 1 Tr 2-225. 08222 Terrassa. Catalonia. Spain, e-mail: romeral@eel.upc.edu
}

\begin{abstract}
This paper presents and analyzed short circuit failures for Permanent Magnet Synchronous Motor (PMSM). The study includes stated and dynamic condition for experimental and simulation test. The stator current is analyzed by means of higher order spectral analysis (HOSA). HOSA techniques are used for stator current analysis under stable conditions is power frequency spectrum density (PSD); and Multiple Signal Classification (MUSIC), and bispectrum are used under dynamics conditions. Therefore, it is possible to improve the accuracy and efficiency of technique. Experimental results validate the analysis and demonstrate for HOSA can be applied to detect and identify short circuit failures in synchronous machines.
\end{abstract}

Index Terms - PMSM drive, Short Circuit, Higher Order Spectral Analysis, HOSA, power frequency spectrum density, PSD, Bispectrum, Multiple Signal Classification, MUSIC.

\section{INTRODUCTION}

Synchronous-type electric motor drives are now extensively used in many industrial applications requiring high system reliability, high efficiency and extended highspeed operation. In many of these critical drive applications, it is required the drive comprising the motor and the converter, under fault conditions, to operate stably and meet base drive needs for a period of time before the system can be (self) - repaired [1]

Stator or armature faults are usually related to insulation failure. In common parlance, they are generally known as phase-to-ground or phase-to-phase faults. It is believed that these faults start as undetected turn-to-turn faults that finally grow and culminate into major ones [2].

Short circuits are the most important failures in PMSM [35], not only to be the main reason of the motor damages, but also for the problems these failures can provoke in high security applications. These applications require an earlier fault detection, in order to isolate the failure and assure fault tolerant post control of the drive.

There are many techniques to detect turn-to-turn faults, the majority of them based on the analysis of stator voltages and currents, axial flux and d-q current and voltage component. Toliyat $[6,7]$ and Penman [8] have described the operation of induction motor drives under the conditions of loss of one phase, broken bars and shorted stator turns using winding functions. However, the models they considered did not include either saturation or control interactions. From years ago, the failure detection in a motor is studied by analyzing the stator current harmonic by means of FFT [9]. But, FFT cannot be applied in no-stationary signals $[1,10]$. Fortunately, signal processing theories provide several algorithms for applications with no-stationary signals [11].

Joint time-frequency analysis is a novel approach in the motor diagnosis applications. Successful use of these techniques requires understanding of their respective properties and limitations [12-14].

The higher order spectral analysis (HOSA) has been considerable interest to researchers in the signal processing and this interest has recently been extended to the condition monitoring $[15,16]$. HOSA requires no a priori data for fault detection and quantification. The disadvantages of HOSA is that has a high computational overhead, and their complex interpretation [16, 17]. However, the model order and the processing time can be reduced using filtering and frequency decimating techniques as have described in [18-20].

This paper suggest that is possible to study and identify short circuits in the windings of the PMSM determined by means of HOSA as PSD, MUSIC and bispectrum in the whole operation range. The motor was experimental tested and simulation with a short circuit in 4,8 and 12 turns for speed between $600 \mathrm{rpm}$ and $6000 \mathrm{rpm}$. Experimental results were compared with those obtained from a healthy machine, and conclusions are done.

\section{Higher ORDER SPECTRAL ANALYSIS (HOSA)}

They are mainly based on statistical procedure and they compute the power frequency spectrum density (PSD). These methods are classified in non-parametric, parametric and subspace techniques $[16,19,20]$. The non-parametric methods have the same problems compared to the classical FFT concerning the frequency resolution. Due to a priori knowledge, the parametric methods have improved performances (Burg, Yule-Walker, covariance and modified covariance). Then, the parametric methods do not validate the traditional assumption that the data outside the window are equal to zero and their performances are affected by the signal-to-noise ratio. The subspace methods are known as high resolution methods as MUSIC, bispectrum or trispectrum which detect frequencies with low signal-to-noise ratio [19]. 
Readers are directed to $[15,16]$ for a rigorous treatment of HOSA fundamentals and subsequent derivations, however, given a discrete time signal $x(n)$, the discrete Fourier transform (DFT) of is defined to be:

$$
X(f)=\sum_{n=-\infty}^{\infty} x(n) e^{-2 j \pi n}
$$

The well-known second-order measure, the power spectral density (PSD) $\mathrm{P}(f)$ of $\mathrm{x}(n)$ can be defined in terms of $\mathrm{X}(f)$ as:

$$
P(f)=E\left[X(f) X^{*}(f)\right]
$$

Where $\mathrm{E}[]$ is the statistical expectation, or average, and $\mathrm{X}^{*}(f)$ is the complex conjugate of $\mathrm{X}(f)$. The PSD is a linear transform and is a function of the frequency index $f$.

Extending these definitions to third-order measures gives rise to the bispectrum defined in (3).

$$
B\left(f_{1}, f_{2}\right)=E\left[X\left(f_{1}\right) X\left(f_{2}\right) X *\left(f_{1}+f_{2}\right)\right]
$$

From (3) it may be seen that, unlike the PSD, the bispectrum are functions of more than one frequency index and, further, it may also be seen that they are complex quantities, i.e., they contain both magnitude and phase information about the original time series $[16,17]$.

\section{A) Multiple Signal Classification (MUSIC)}

Multiple Signal Classification (MUSIC) method associates the noise to the minimum eigenvalue of the noise variance allowing low signal-to-noise frequency components estimation and the frequency estimation is based on the orthogonality of the signal vectors to the noise eigenvectors $[18,19]$.

The subspace methods assume that data $x[n]$ can be represented by $m$ complex sinusoids in a noise $e[n][15,18]$ :

$$
x[n]=\sum_{i=1}^{m} A_{i} e^{j 2 \pi f_{i} n}+e[n] \quad A_{i}=\left|A_{i}\right| e^{j \phi_{i}}
$$

Where $\mathrm{n}=0,1,2, \ldots N-1, N$ is the number of sample data, $f_{i}$, $\left|A_{i}\right|$ and $\varphi_{\mathrm{i}}$ are the frequency, the magnitude and the random phase of $i^{\text {th }}$ complex sinusoid respectively, $e[n]$ is a sequence with a zero mean white noise.

The method known as Pisarenko harmonic decomposition (PHD) shows that the frequencies of the sinusoids can be obtained from the eigenvector corresponding to the smallest eigenvalue of the autocorrelation matrix. When $e[n]$ is with zero mean white noise and a variance $\sigma^{2}$, the autocorrelation matrix $\boldsymbol{R}$ of $x[n]$ can be represented by:

$$
\begin{aligned}
& R=\sum_{i=1}^{m}\left|A_{i}\right|^{2} e\left(f_{i}\right) e^{H}\left(f_{i}\right)+\sigma^{2} I \\
& e\left(f_{i}\right)=\left[1 e^{j 2 \pi f_{i}} e^{j 4 \pi f_{i}} \ldots e^{j 2 \pi(N-1) f_{i}}\right]^{T}
\end{aligned}
$$

The exponent $\boldsymbol{H}$ is the Hermitian transpose and $\mathrm{I}$ is the identity matrix. It is seen that the autocorrelation matrix $\boldsymbol{R}$ is composed of the sum of signal and noise autocorrelation matrices:

$$
R=R_{s}+\sigma^{2} I
$$

Pisarenko observed that the noise variance corresponds with the smallest eigenvalue of $\boldsymbol{R}$ and that the frequencies of the complex sinusoids can be estimated by using the orthognality of the signal and noise subspaces, that is:

$e\left(f_{i}\right)^{H} V(m+1)=0, i=1,2 \ldots, m$

With $\mathbf{V}(m+1)$ the noise eigenvector in a matrix $\mathbf{R}$ with the dimension $(m+1) \times(m+1)$.

MUSIC approach has better performances than Pisarenko method because of the introduction of averaging via the extra noise eigenvectors $(k=m+1, m+2, \ldots, M)$. The averaging reduces the statistical fluctuations present in the Pisarenko pseudo-spectrum which are due to the errors in estimating the autocorrelation matrix. Then, the MUSIC pseudo-spectrum of space vector is defined as:

$$
P^{\text {MUSIC }}(f)=\frac{1}{\sum_{k=m+1}^{M}\left|e(f)^{H} V_{k}\right|^{2}}
$$

Where $e_{i}\left(f_{i}\right)^{H} V_{k}=0, k=m+1, \ldots, M$. It has the largest peaks at the principal components and these frequencies are in the bandwidth $\left[0, F_{s} / 2\right]$ for a signal sampled with $F_{s}$ sampling frequency.

The MUSIC method takes a long computation time to find more frequencies by increasing the order of the frequency signal dimension. To solve this problem, an algorithm based on zooming in a specific frequency range is proposed with MUSIC in order to improve the diagnosis performances to extract frequencies $[18,19]$. The Zoom-MUSIC algorithm is a technique to make the MUSIC faster and more accurate in extracting frequencies in a specified bandwidth [18-20].

\section{WORKBENCH OF SIMULATION AND EXPERIMENTAL}

The motors under analysis have been a PMSM of $6000 \mathrm{rpm}$ nominal speed, $2.3 \mathrm{Nm}$ nominal torque, and 3 poles pair [10]. Simulations and experiments have been carried out for motors with 4,8 and 12 short turns of stator phase winding; the motors were driven at nominal, medium (4500 and $3000 \mathrm{rpm}$ ) and low (1500 and $600 \mathrm{rpm}$ ) speed. Both, the simulations and the experiments were carried out under stationary and no-stationary conditions.

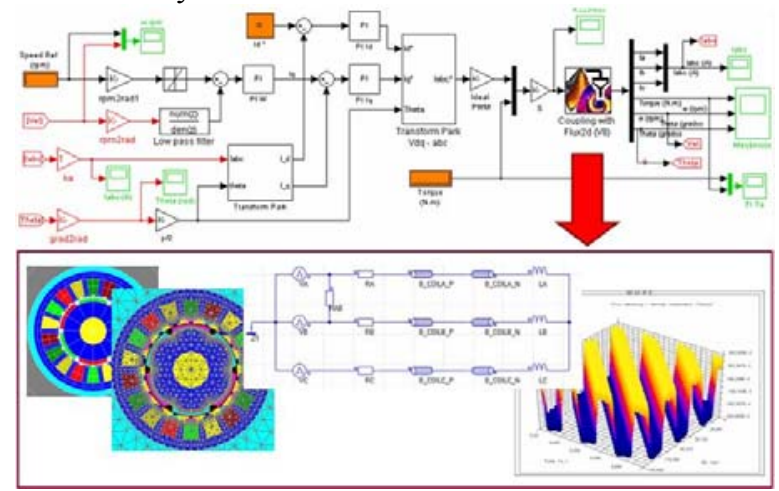

Fig. 1. Schematic of the finite element analysis (FEA) for a PMSM

Finite element analysis (FEA) is proposed for the simulation of electrical machines with short circuit fault. FEA reveals itself like an accurate an easy method to determine the interaction between non-linear effects. PMSM in a healthy state and with short circuit turns are simulated at different 
working points using a two-dimensional (2-D) finite-element analysis software (FLUX 2D) [21]. The PMSM workbench for FEA analysis is shown in

Fig. 1.

The Higher Order Spectral Analysis Toolbox with Matlab is used to compute the HOSA, PSD, MUSIC and Bispectrum [15].

\section{SIMULATION AND EXPERIMENTAL RESULTS}

Some experiments have been carried out for different motors with shorted turns. The analysis is made for stable condition of speed and constant torque. The results of machine with short circuit are compared with those obtained from healthy machines.

\section{A. Stable State (speed and constant torque)}

The spectral analysis of stator current is made using Power Spectral Density (PSD) or Multiple Signal Classification (MUSIC). These methods allow to identify the most representative frequencies filtering the Gaussian noise and giving priority the frequencies of higher value, that in this case are the main frequency of stator and failure frequency. This way, the failure detection is assured and the resolution for interest frequencies is improved.

Fig. 2 and Fig. 3 show MUSIC of stator current for 1500 and $6000 \mathrm{rpm}$ respectively. For $6000 \mathrm{rpm}$, the main harmonic $300 \mathrm{~Hz}$ and failure harmonic at $900 \mathrm{~Hz}$ for PMSM with short circuit are observed, the others harmonics like $1^{\text {st }}, 5^{\text {th }}$ and $7^{\text {th }}$ of machines are covering by the sweeping of spectrum and it can not seen. The last harmonics normally are present when it is analyzed by means of classic MCSA, therefore, MUSIC has filtered these harmonics and has centred the analysis in the main harmonic of stator and $9^{\text {th }}$ harmonic.

The experimental results are show in Fig. 4 and Fig. 5 for MUSIC at 1500 and $6000 \mathrm{rpm}$ respectively. MUSIC shows a high value for $9^{\text {th }}$ harmonic for PMSM with short circuit. For $6000 \mathrm{rpm}$, MUSIC centers the analysis in the main harmonic of stator $(300 \mathrm{~Hz})$ and the failure harmonic $(900 \mathrm{~Hz})$ for PMSM with short circuit. However, for healthy machines the $9^{\text {th }}$ harmonic is filtered. In this case of healthy machine the MUSIC analysis takes another frequency from greater amplitude like $15^{\text {th }}$ and $21^{\text {st }}$ harmonic. Those harmonics have high amplitude in healthy machine by the constructive form: concentrated winding, slot number by pole and phase (2); in addition, in the case of simulation the slot inclination is not considered.

Also, Note that in case of healthy machine at $1500 \mathrm{rpm}$, MUSIC takes the main harmonic and $10^{\text {th }}$ harmonic like main values and not $9^{\text {th }}$ harmonic. This is due to $9^{\text {th }}$ and $10^{\text {th }}$ harmonics have similar values. But in any case, the failure condition to low frequencies is possible to identify.

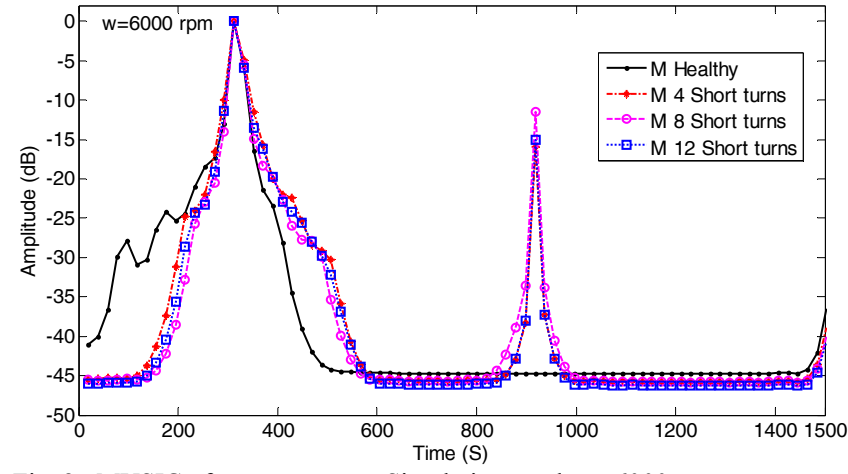

Fig. 2. MUSIC of stator currents, Simulation results at $6000 \mathrm{rpm}$

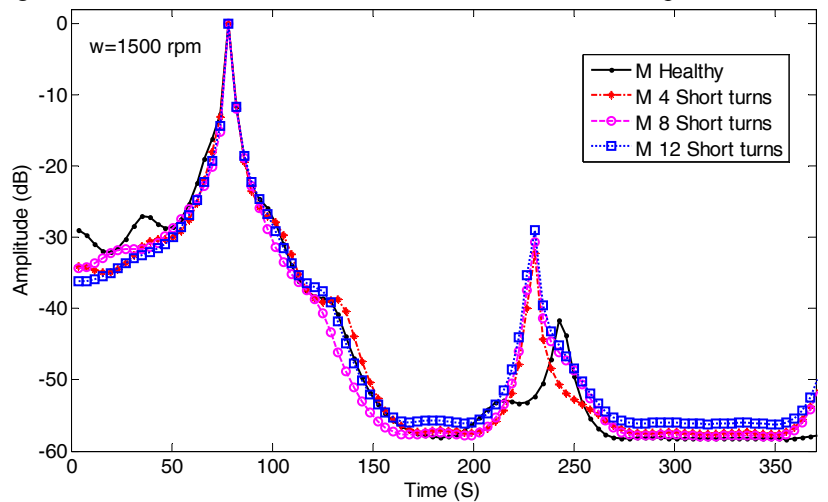

Fig. 3. MUSIC of stator currents, Simulation results at $1500 \mathrm{rpm}$

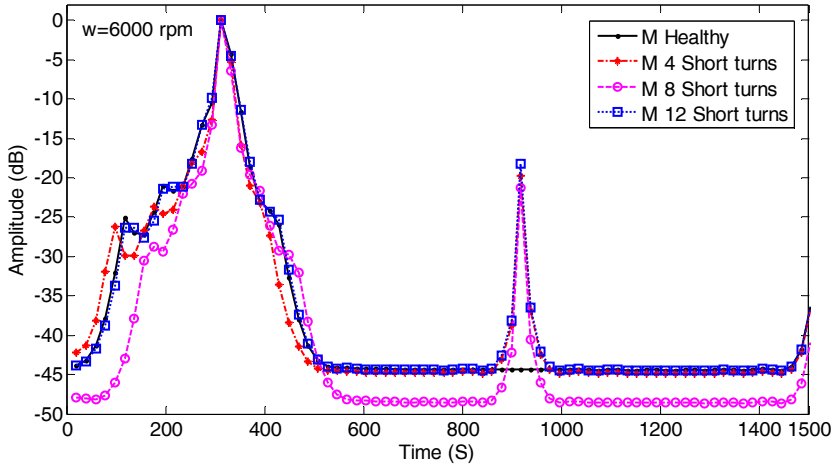

Fig. 4. MUSIC of stator currents, Experimental results at $6000 \mathrm{rpm}$

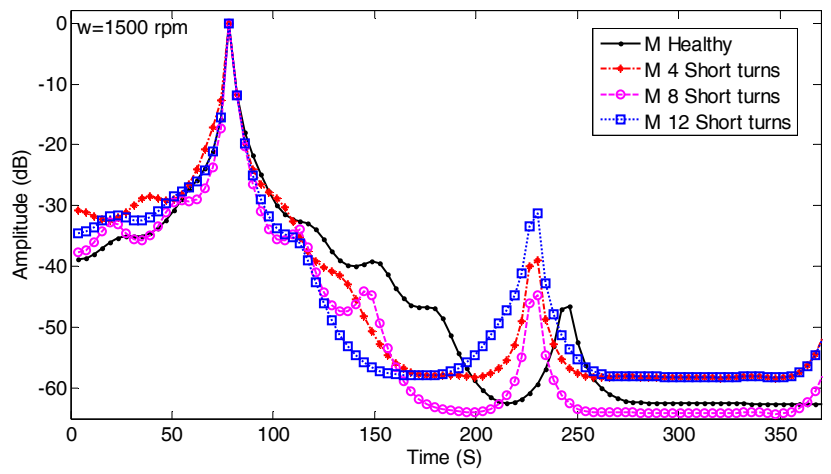

Fig. 5. MUSIC of stator currents, Experimental results at $1500 \mathrm{rpm}$

Simulation results of PSD are show in Fig. 6 and Fig. 7 for $6000 \mathrm{rpm}$ and $1500 \mathrm{rpm}$ respectively. The difference of amplitude is high in $9^{\text {th }}$ harmonic between healthy and faulty machines. This difference is greater than the MCSA classic method reported before $[4,5]$. PSD shown an upper value of 
$30 \mathrm{~dB}$ for PMSM with short circuit and maintain this difference even for low speed where classic MCSA method presents trouble.

Fig. 8 and Fig. 9 show PSD for $6000 \mathrm{rpm}$ and $1500 \mathrm{rpm}$ respectively. They have been taken from experimental results. They approve the simulation and validate the method for short circuit detection. Although the resolution is good around of failure frequency, in the rest of spectrum isn't as much, ripple amplitude with range of $\pm 5 \mathrm{~dB}$ can be seen.

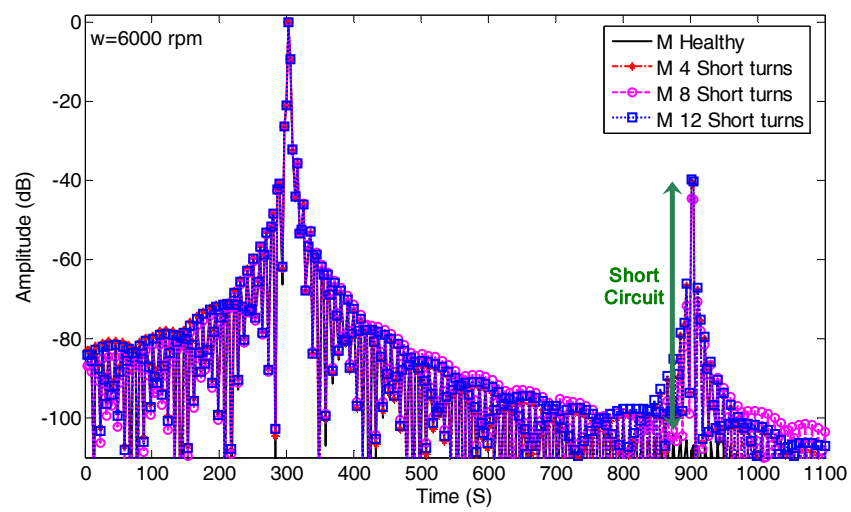

Fig. 6. PSD of stator currents, Simulation results at $6000 \mathrm{rpm}$

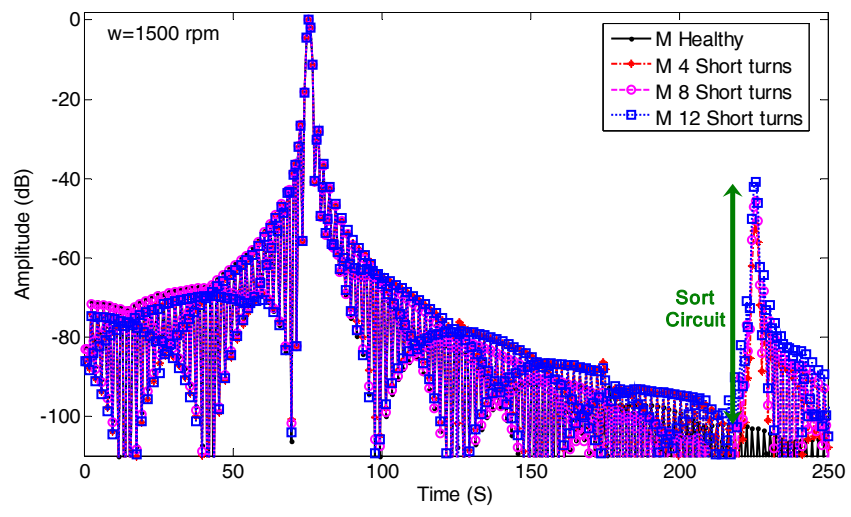

Fig. 7. PSD of stator currents, Simulation results at $1500 \mathrm{rpm}$

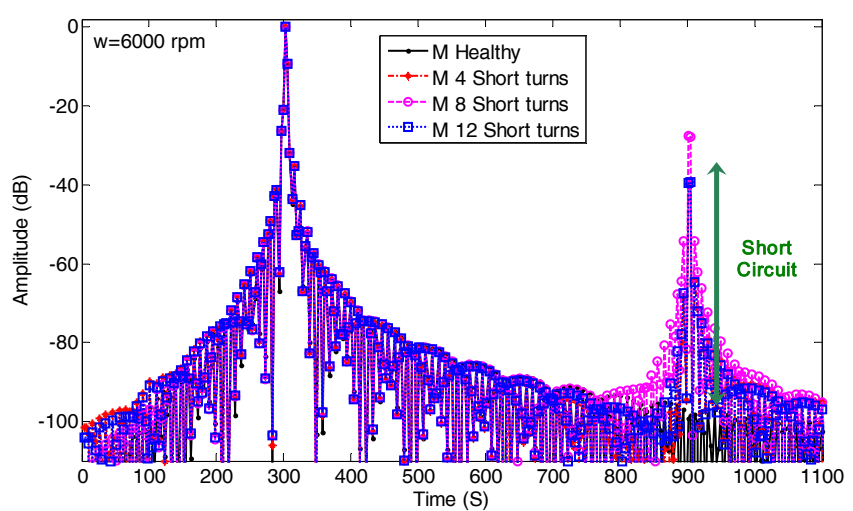

Fig. 8. PSD of stator currents, Experimental results at $6000 \mathrm{rpm}$

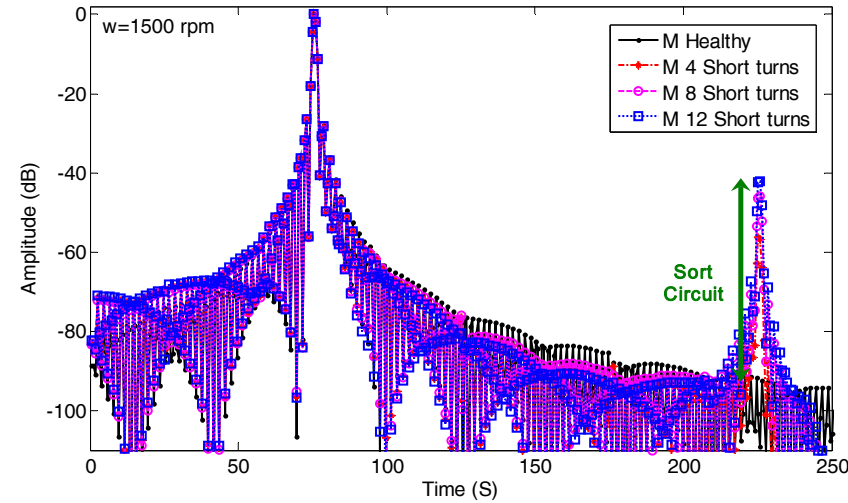

Fig. 9. PSD of stator currents, Simulation results at $1500 \mathrm{rpm}$

In relation at MCSA standard, the PSD and MUSIC methods allow to improve the resolution of short circuit harmonic; and they eliminate the noise of signal efficiently due to external sources or even internal sources of less interest, these are: unbalanced tension, network harmonics, sub harmonics, modulation of inverter, leak of isolation, vibrations, speed changes, ripple torque, stator and rotor magnetic loss, saturation, etc.

The advantage of MUSIC and PSD methods is that estimated spectrum is very clean and flat (without noise) in frequency range of high interest (outstanding). On the other hand, HOSA allows estimated spectrum for different methods from third and fourth order. Thus, better resolution in the most notable frequencies at a proportional computational cost can be established. Although the computational cost can be decreased if the analysis is centered in interest frequencies range like several methods reported by Zoom-FFT and ZoomMUSIC [18-20].

Biespectrum looks for sum and multiplication relationships of two interest frequencies that in this case are main frequency of stator and failure frequency. Fig. 10 and Fig. 11 show bispectrum for healthy and fault PMSM. This simulation dates are taken the whole stator current. For PMSM with short circuit are two ovals in the graph. The internal ovals correspond to main frequency $(300 \mathrm{~Hz})$ of stator current and external oval is around $900 \mathrm{~Hz}$ that is the failure frequency.

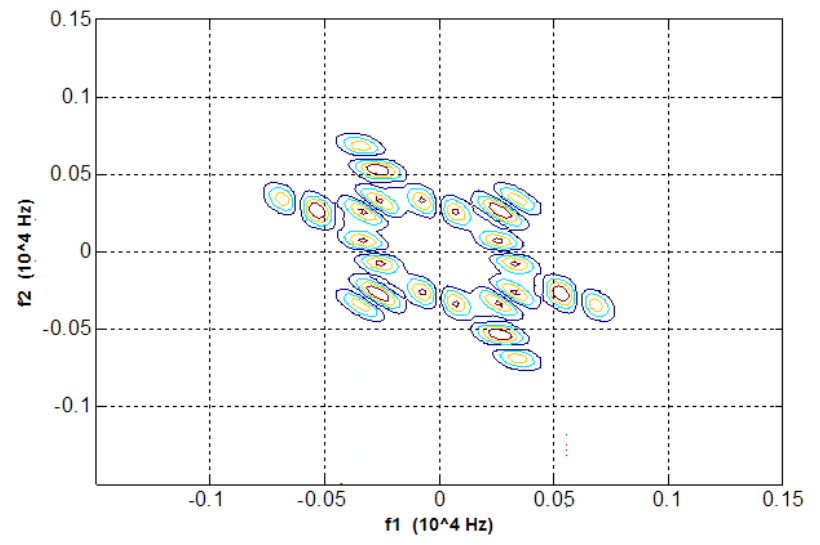

Fig. 10. Bispectrum of healthy PMSM, Simulation results at $6000 \mathrm{rpm}$ 


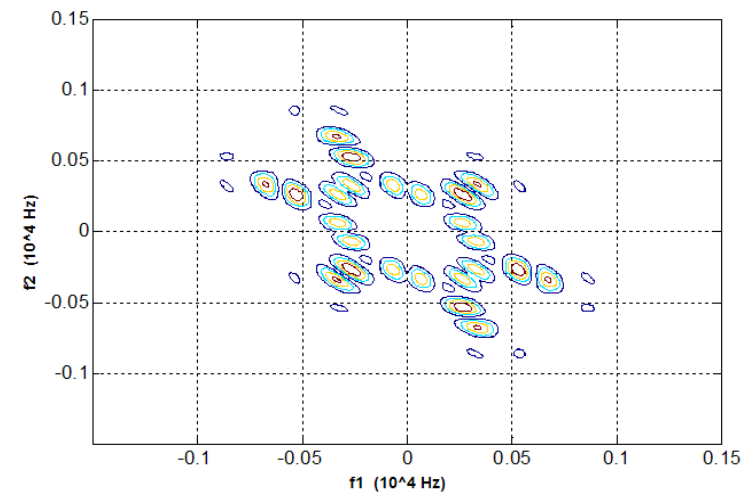

Fig. 11. Bispectrum of PMSM with 12 short circuit turns, Simulation results at $6000 \mathrm{rpm}$

Bispectrum allows viewed interaction of harmonics of machine and the same as PSD and MUSIC centered the analysis in main frequencies assigning them bigger value. Thus, Bispecturm maintains interest frequency as the failure frequency of short circuit for the whole operation range of machine. Similar results for $600 \mathrm{rpm}, 3000 \mathrm{rpm}$ and $4500 \mathrm{rpm}$ has been obtained.

Bispectrum can be used for current analysis in dynamic condition of speed or torque variation. This even would reinforce the ability of method to show the interaction of frequencies and magnitude change at the same time.

\section{B. Speed Change (constant torque)}

The PMSM usually operates to different speeds and torque conditions. Under these dynamic conditions, the short circuit detection can be carried out by means of analysis of time frequency of the stator current. As it could be seen in the previous section, the proposed method is able to perform fault detection at a low speed, constant conditions. Next, it is demonstrated that it is also useful for medium and high speeds under variable conditions.

From Fig. 12 to Fig. 14 show stator current bispectrum for PMSM with short circuit. These are simulation and experimental results for speed changes at 1500 to $1000 \mathrm{rpm}$. Therefore, the short circuit frequency in the speed change is reinforces with interaction with other harmonics and make more visible the failure. Mainly the interaction with main harmonic $(75 \mathrm{~Hz})$ and $5^{\text {th }}$ harmonic $(125 \mathrm{~Hz})$ can be seen. Although others frequencies are present in the graph are less notable.

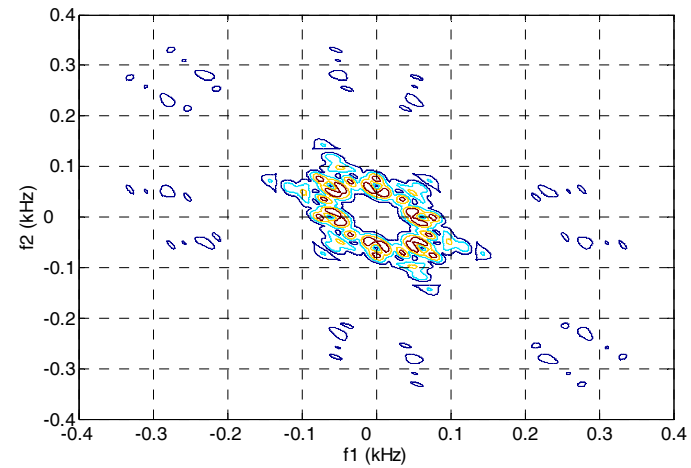

Fig. 12. Bispectrum of stator currents in PMSM with 8 short circuit turns, Simulation results for speed change from 1500 to $1000 \mathrm{rpm}$

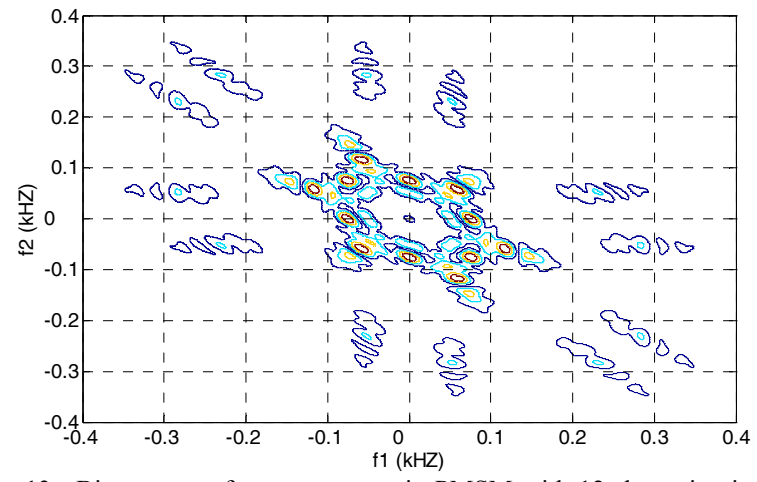

Fig. 13. Bispectrum of stator currents in PMSM with 12 short circuit turns, Simulation results for speed change from 1500 to $1000 \mathrm{rpm}$

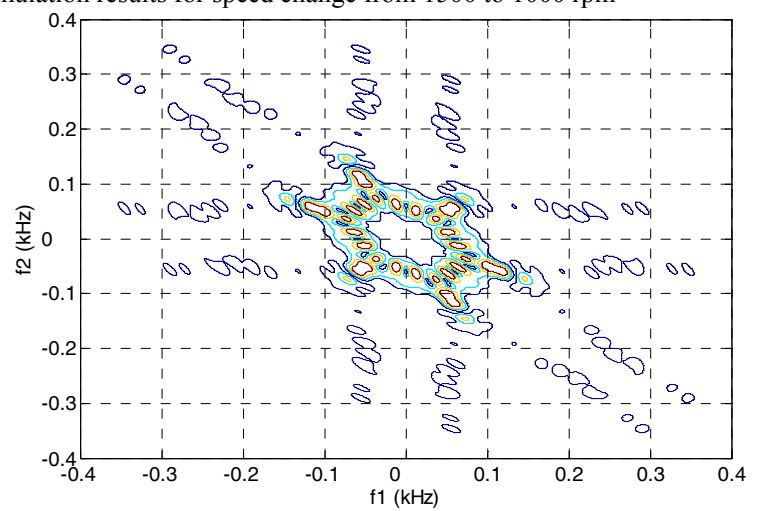

Fig. 14. Bispectrum of stator currents in PMSM with 12 short circuit turns, Experimental results for speed change from 1500 to $1000 \mathrm{rpm}$

For speeds of $6000 \mathrm{rpm}, 4500 \mathrm{rpm}, 3000 \mathrm{rpm}$ and $600 \mathrm{rpm}$ similar results are obtained and therefore, only the results for less favorable condition are shown.

The proposed method display excellent ability to short circuit detect in constant and dynamic conditions.

The analysis by means of bispectrum allows composed a powerful tool for fault detection of short circuit in PMSM. This method can be applied for others failure types as eccentricity and demagnetization where the harmonics of interest are $1^{\text {st }}$ and $5^{\text {th }}$ harmonics.

The computational cost of HOSA techniques as PSD, MUSIC or bispectrum can be reduced significantly if it is applied only for interest frequency range as several authors show for zoom-FFT and Zoom-MUSIC [18-20].

\section{CONCLUSIONS}

This paper presents the short circuit detection in a PMSM by means of Higher Order Spectral Analysis (HOSA).

PSD and MUSIC show feature signature differentiate between healthy and faulty conditions, as well as between degrees of fault for stated state. The previous methods maximize the difference for short circuit harmonic and their resolution is greater in relation with classic MCSA. Also, PSD and MUSIC can detect short circuit for the whole speed range. These methods can be used for preventive maintenance where the test in controlled and known conditions can be carried out.

The computational cost of PSD, MUSIC or Bispectrum algorithm can be reduced with zoom techniques that make the 
method faster and more accurate in extracting frequencies in a specified frequency range.

\section{REFERENCES}

[1] J. Rosero, J. Cusido, A. Garcia, J. A. Ortega, and L. Romeral, "Broken bearings and eccentricity fault detection for a permanent magnet synchronous motor," in The 32nd Annual Conference of the IEEE Industrial Electronics Society, IECON06, Paris FRANCE, 2006, pp. 964-969.

[2] J. A. Haylock, B. C. Mecrow, A. G. Jack, and D. J. Atkinson, "Operation of fault tolerant machines with winding failures," IEEE Transactions on Energy Conversion, vol. 14, p. 1490, 1999.

[3] S. Nandi, H. A. Toliyat, and X. Li, "Condition Monitoring and Fault Diagnosis of Electrical Motors - A Review," IEEE Transactions on Energy Conversion, vol. 20, p. 719, 2005.

[4] J. Rosero, J. Cusido, L. Romeral, and J. A. Ortega, "Detection of Stator Short Circuits in PMSM by mean of joint Time-Frequency Analysis," in The 6th IEEE International Symposium on Diagnostics for Electric Machines, Power Electronics and Drives, IEEE SDEMPED, Cracow, Poland, 2007, pp. 420-425.

[5] J. Rosero, O. Almonacid, M. Amaya, and L. Romeral, "Simulation and Fault Detection of Short Circuit Winding in a Permanent Magnet Synchronous Machines (PMSM)," in ISEF 2007 - XIII International Symposium on Electromagnetic Fields in Mechatronics, Electrical and Electronic Engineering, Prague, Czech Republic, 2007.

[6] H. A. Toliyat, S. P. Waikar, and T. A. Lipo, "Analysis and simulation of five-phase synchronous reluctance machines including third harmonic of airgap MMF," IEEE Transactions on Industry Applications, vol. 34, pp. 332-339, 1998.

[7] H. A. Toliyat and T. A. Lipo, "Transient analysis of cage induction machines under stator, rotor bar and end ring faults," IEEE Transactions on Energy Conversion, vol. 10, pp. 241-247, 1995.

[8] G. M. Joksimovic and J. Penman, "The detection of inter-turn short circuits in the stator windings of operating motors," IEEE Transactions on Industrial Electronics, vol. 47, pp. 1078-1084, 2000 .

[9] J. Rosero, L. Romeral, J. Cusido, A. Garcia, and J. A. Ortega, "On the short-circuiting fault detection in a PMSM by means of stator current transformations," in The 38th IEEE Power Electronics Specialists Conference, PESC07, Orlando, Florida, USA, 2007.

[10] J. Rosero, J. Cusido, A. Garcia, J. A. Ortega, and L. Romeral, "Study on the Permanent Magnet Demagnetization Fault in Permanent Magnet Synchronous Machines," in The 32nd Annual Conference of the IEEE Industrial Electronics Society, IECON06, Paris - FRANCE, 2006, pp. 879-884.

[11] N. Arthur and J. Penman, "Condition monitoring with non-linear signal processing," in IEE Colloquium on Non-Linear Signal and Image Processing (Ref. No. 1998/284), , 1998, pp. 4/1-4/5.

[12] R. Yan and R. X. Gao, "Hilbert\&ndash;Huang Transform-Based Vibration Signal Analysis for Machine Health Monitoring," IEEE Transactions on Instrumentation and Measurement,, vol. 55, pp. 2320-2329, 2006.

[13] J. Cusido, J. A. Rosero, J. A. Ortega, A. Garcia, and L. Romeral, "Induction Motor Fault Detection by using Wavelet decomposition on dq0 components," in IEEE International
Symposium on Industrial Electronics, ISIE06, Montréal, Québec, Canada, 2006, pp. 2406-2411, vol. 3.

[14] Z. Yuping, "Hilbert-Huang Transform and Marginal Spectrum for Detection of Bearing Localized Defects," in The Sixth World Congress on Intelligent Control and Automation, WCICA 2006. , Dalian, China, 2006, pp. 5457-5461.

[15] A. Swami, J. M. Mendel, and C. Nikias, Higher Order Spectral Analysis Toolbox for use with MATLAB: United Signals \& Systems, Inc., 1998.

[16] N. Arthur and J. Penman, "Induction machine condition monitoring with higher order spectra," IEEE Transactions on Industrial Electronics, vol. 47, pp. 1031-1041, 2000.

[17] A. C. McCormick and A. K. Nandi, "Bispectral and trispectral features for machine condition diagnosis," IEE Proceedings Vision, Image and Signal Processing, vol. 146, pp. 229-234, 1999.

[18] S. H. Kia, H. Henao, and G. A. Capolino, "A High-Resolution Frequency Estimation Method for Three-Phase Induction Machine Fault Detection," IEEE Transactions on Industrial Electronics, vol. 54, pp. 2305-2314, 2007.

[19] S. H. Kia, H. Henao, and G. A. Capolino, "Zoom-MUSIC frequency estimation method for three-phase induction machine fault detection," in IECON 2005. 31st Annual Conference of Industrial Electronics Society. IEEE, Nort Carolina, USA, 2005, pp. 2603-2608.

[20] L. A. Pereira, D. Fernandes, D. S. Gazzana, F. B. Libano, and S Haffner, "Application of the Welch, Burg and MUSIC Methods to the Detection of Rotor Cage Faults of Induction Motors," in Transmission \& Distribution Conference and Exposition: Latin America, 2006. TDC '06. IEEE/PES, 2006, pp. 1-6.

[21] CEDRAT, CAD package for electromagnetic and thermal analysis using finite elements, User's guide. Flux 2D. France, 2005, www.cedrat.com 